\title{
Primary mucinous adenocarcinoma of the vulva: A case report and review of the literature
}

\author{
YANXIA SUI ${ }^{1,2^{*}}$, JUNKAI ZOU $^{1 *}$, NASRA BATCHU ${ }^{1}$, SHULAN LV $^{1}$, \\ CHAO SUN ${ }^{1}$, JIANG DU ${ }^{1}$, QING WANG ${ }^{1}$, QING SONG ${ }^{1,3}$ and QILING LI ${ }^{1}$ \\ Departments of ${ }^{1}$ Obstetrics and Gynecology and ${ }^{2}$ Pathology, First Affiliated Hospital of Xi'an Jiaotong University, Xi'an, \\ Shaanxi 710061, P.R. China; ${ }^{3}$ Cardiovascular Research Institute, Morehouse School of Medicine, Atlanta, GA 30310, USA
}

Received June 4, 2015; Accepted November 26, 2015

DOI: $10.3892 / \mathrm{mco} .2016 .766$

\begin{abstract}
Primary vulvar cancer is a rare disease with an incidence of 2-3 per 100,000 women. The vast majority of vulvar carcinomas are of the squamous cell type (90\%). Primary vulvar adenocarcinomas rank among the rare gynecological malignancies. We herein present a case of a vulvar mass near the vaginal orifice, the biopsy of which revealed a mucinous adenocarcinoma. Local excision was performed, followed by postoperative chemotherapy. The patient was asymptomatic and developed no recurrence during the 2 years of follow-up after surgery and chemotherapy. We consider local excision, with or without chemotherapy, to be an effective therapeutic approach to this type of tumor. However, further studies are required to support our conclusions for early-stage vulvar mucinous adenocarcinoma.
\end{abstract}

\section{Introduction}

Primary vulvar cancer is a rare disease, with an incidence of 2-3 per 100,000 women. Traditionally, primary vulvar cancer affects elderly women (median age, 65-70 years) and the vast majority of tumors are squamous cell carcinomas (90\%) (1). Primary vulvar adenocarcinomas are rare and their histogenesis has not been fully elucidated; they mainly include extramammary Paget's disease, sweat gland carcinomas and breast-like

Correspondence to: Dr Qiling Li, Department of Obstetrics and Gynecology, First Affiliated Hospital of Xi'an Jiaotong University, 277 Yanta West Road, Xi'an, Shaanxi 710061, P.R. China

E-mail: liqiling@mail.xjtu.edu.cn

*Contributed equally

Abbreviations: PAS, periodic acid-Schiff; AB, alcian-blue; CK, cytokeratin; CAM, cellular adhesion molecule; ER, estrogen receptor; PR, progesterone receptor; GCDFP, gross cystic disease fluid protein; TIF, transcription intermediary factor; CA, carbohydrate antigen; PET-CT, positron emission tomography-computed tomography; CEA, carcinoembryonic antigen; IHC, immunohistochemistry

Key words: adenocarcinoma, vulvar carcinoma, management adenocarcinomas of the vulva $(2,3)$. Primary vulvar mucinous adenocarcinoma, in particular, is an extremely rare entity, with only one case reported to date (4). The aim of this report was to describe another case of mucinous adenocarcinoma arising from the vulva and discuss its clinical management.

\section{Case report}

A Chinese woman, aged 43 years, gravida 3, para 2, presented with a 1-month history of a pruritic, painless vulvar mass. On gynecological examination, a $1.5-\mathrm{cm}$ soft, non-tender gray mass was identified adjacent to the hymen on the perineal body. Bioptic excision of this mass was performed at another hospital and the pathological examination revealed an invasive, moderately differentiated mucinous adenocarcinoma (Fig. 1A). On immunohistochemical analysis, the tumor cells were diffusely positive for periodic acid-Schiff (PAS), alcian blue (AB), cytokeratin (CK) 7 and cellular adhesion molecule (CAM) 5.2, and focally positive for p16 (Fig. 1B-F). The tumor cells were negative for estrogen receptor (ER), progesterone receptor (PR) and gross cystic disease fluid protein (GCDFP) 15, thereby excluding mammary gland and endometrial carcinoma. The tumor was also negative for carbohydrate antigen (CA) 125, excluding ovarian cancer. The tumor stained negatively for CK20, villin and CA19-9, excluding adenocarcinoma of the gastrointestinal tract. The tumor stained negatively for transcription intermediary factor (TTF) 1 and napsin A, excluding lung adenocarcinoma. Therefore, the diagnosis of primary mucinous adenocarcinoma of the vulva was confirmed. An extensive workup, including chest X-ray, abdominal and pelvic ultrasound, cervical Pap smear and systemic positron emission tomography-computed tomography (PET-CT) did not reveal any other local genital or systemic cancer. In particular, colonoscopy and gastroscopy examination were both negative, excluding adenocarcinoma of the gastrointestinal tract (5). The carcinoembryonic antigen (CEA) and CA-125 levels were not increased. A diagnosis of stage I primary mucinous adenocarcinoma of the vulva was made, according to the classification of the International Federation of Gynecology and Obstetrics. The patient's general health was good and her routine laboratory examinations were considered satisfactory for her age.

A local resection of the vulvar neoplasm without lymph node dissection was recommended. The postoperative pathological 

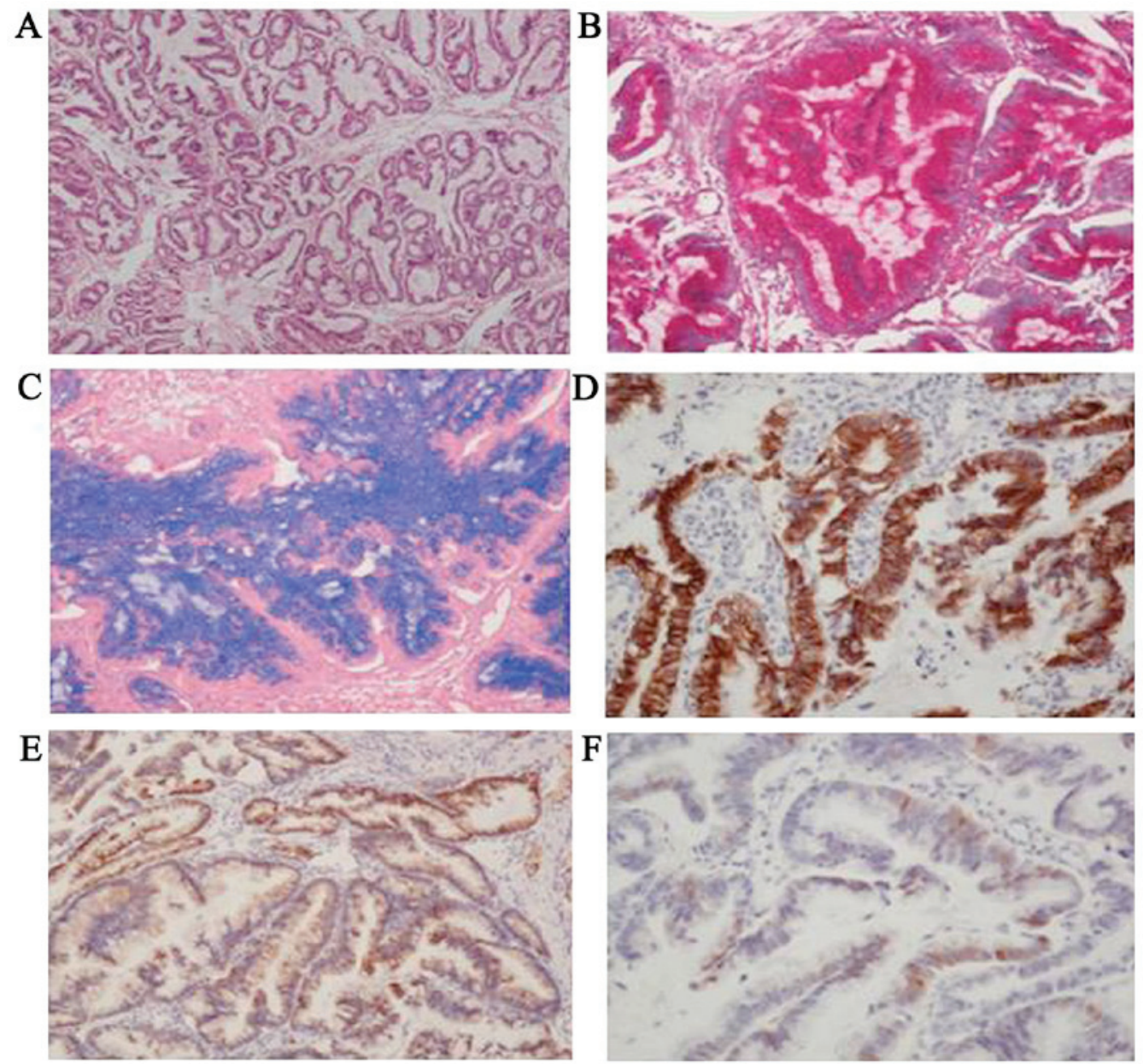

Figure 1. Mucinous adenocarcinoma of the vulva. (A) The cancer tissue exhibited an adenoid structure. The glands were of varying sizes, irregular, with a complex, incomplete structure. Secreted mucus was found in the gland cavity and mesenchyme. The mesenchyme consisted of loose fibrous connective tissue with scattered inflammatory cells (hematoxylin and eosin staining; magnification, x40). (B) Positive periodic acid-Schiff staining [immunohistochemistry (IHC); magnification, x200]. (C) Positive alcian blue staining (IHC; magnification, x200). (D) Positive cytokeratin 7 staining (IHC; magnification, x200). (E) Positive cellular adhesion molecule 5.2 staining (IHC; magnification, x200). (F) Positive p16 staining (IHC; magnification, x200).

examination revealed an invasive, moderately differentiated mucinous adenocarcinoma, with clear resection margins of the surgical specimen. With the patient's informed consent, she then underwent systemic venous chemotherapy. The combined chemotherapy consisted of paclitaxel $\left(150 \mathrm{mg} / \mathrm{m}^{2}\right)$ and carboplatin $\left(220 \mathrm{mg} / \mathrm{m}^{2}\right)$ for a total of four cycles (6). The patient underwent a regular clinical, vulvoscopic and colonoscopic follow-up and she remains alive and cancer-free at 24 months after treatment.

\section{Discussion}

Mucinous adenocarcinoma is a rare variant of sweat gland carcinoma, first described by Mendoza and Helwig in 1971 (7). A total of 76 cases of mucinous adenocarcinoma have been reported in the with three cases on the vulva (Table I). Two of them were primary neuroendocrine differentiated mucinous adenocarcinomas of the vulva $(8,9)$, and only one was a true primary vulvar mucinous adenocarcinoma, which was also the case in our patient. The case report of primary vulvar mucinous adenocarcinoma in the literature was that of an 80-year-old, nulliparous, Caucasian woman, who presented with a 2-month history of a 2-cm, asymptomatic, reddish-blue, well-defined cystic mass on the upper-medial aspect of the right labium majus. The patient underwent radical vulvectomy, with bilateral inguinofemoral lymph node dissection (4).

As regards the clinical management of primary vulvar cancer, up to the 1990s, radical vulvectomy with bilateral inguinofemoral lymphadenectomy was considered the standard therapy. The aim of this intervention was to remove all tissue possibly affected by vulvar cancer, including the bridge of skin between the vulva and the inguinal area. However, this mutilating procedure was associated with severe morbidity, due to the significant psychosexual impairment (10). To avoid overtreatment, increasing efforts to modify surgical management were undertaken. The introduction of radical local excision instead of complete vulvectomy was a major step towards further reduction of surgical complications. No compromise in oncological safety was observed in patients with early-stage disease compared with the control group (1). Considering the significant morbidity of radical lymphadenectomy and the fact that only $25-30 \%$ of the patients present with lymph node metastases at first diagnosis, sentinel node dissection is considered a favorable alternative for patients with a clinically node-negative inguinal area (11). Patients with early-stage disease have a fairly good prognosis with an individualized 


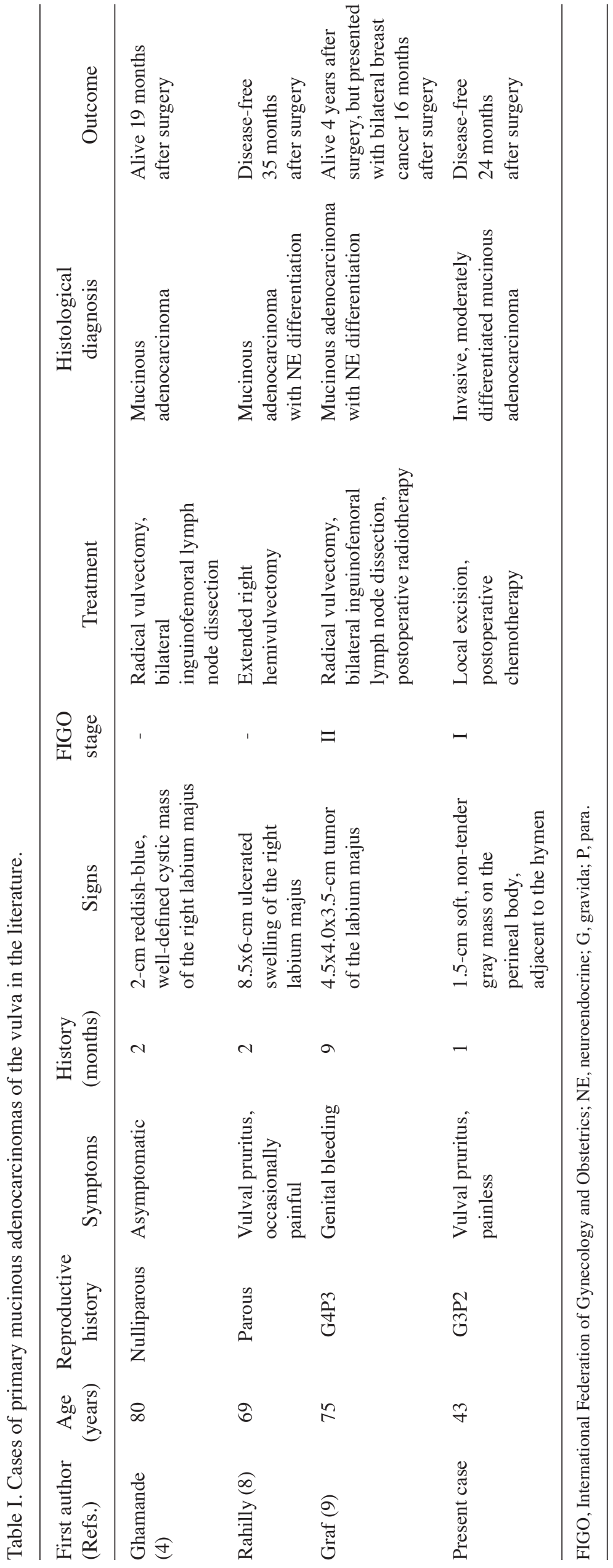


treatment plan and a less radical surgical approach (12). Local tumor resection rather than radical vulvectomy and the implementation of the sentinel technique have decreased therapy-associated morbidity and psychosocial impairment in these patients, while maintaining oncological safety (11).

The clinical management in our case was different from that of the first case of primary vulvar mucinous adenocarcinoma. The reasons were as follows: First, the lesion was adjacent to the hymen on the perineal body in our case. In general, lymphatic metastasis is one of the main pathways of metastasis in gynecological malignant tumors, and a poor prognostic factor (13). As regards vulvar cancer lymph node metastases from the superficial to the deep inguinal and femoral lymph nodes (including the Cloquet's lymph node) and then to the pelvic lymph nodes, the mean incidence is $22-39 \%$ and the lesion size, infiltration depth and stage are closely associated. The case report of vulvar mucinous adenocarcinoma in the literature, the lesion was on the upper medial aspect of the right labium majus, with lymphatic flow mainly to the inguinal lymph nodes; in our case, the tumor was on the perineal body, with lymphatic flow mainly to the pelvic lymph nodes (11). There was no pelvic lymph node metastasis on the systemic PET-CT. Therefore, bilateral inguinofemoral node dissection was not performed. Second, vulvar mucinous adenocarcinoma is rare and its pathway of metastasis has not been elucidated; thus, the main pathway of metastasis may not be lymphogenic. Adjuvant chemotherapy was administered postoperatively to prevent other metastatic pathways $(6,14)$. Finally, the patient's diagnosis in this case was stage IA primary mucinous adenocarcinoma of the vulva and, for tumors with an infiltration depth of $<1 \mathrm{~mm}$, a lymphadenectomy is not considered necessary $(15,16)$. In addition, our patient displayed no signs of pelvic lymph node metastasis. As the lesion was close to the anus and rectum, radical resection would have required removal of those anatomical structures, which would have severely compromised the quality of life of this patient. Therefore, a local tumor resection was performed, followed by chemotherapy.

In the case of vulvar mucinous adenocarcinoma in the literature, the patient remained alive and well 19 months after the surgery. In our case, during the 2 years of follow-up, the patient remained clinically disease-free, without recurrence or treatment-related complications. We consider local excision, with or without chemotherapy, to be an effective therapeutic approach to this type of tumor. However, further studies are required to support our conclusions for early-stage vulvar mucinous adenocarcinoma. Patients with early-stage disease have a fairly good prognosis with an individualized treatment plan and a less radical surgical approach. Therefore, we recommend local excision, with or without chemotherapy, as an effective treatment for early-stage vulvar mucinous adenocarcinoma.

\section{Acknowledgements}

The authors would like to thank to Mrs. Huiting Liu, who revised the manuscript.

\section{References}

1. Woelber L, Kock L, Gieseking F, Petersen C, Trillsch F, Choschzick M, Jaenicke F and Mahner S: Clinical management of primary vulvar cancer. Eur J Cancer 47: 2315-2321, 2011.

2. Kajal B, Talati H, Daya D and Alowami S: Apocrine adenocarcinoma of the vulva. Rare Tumors 5: e40, 2013.

3. Bogani G, Uccella S, Cromi A, Casarin J, Donadello N and Ghezzi F: Primary mammary-like ductal carcinoma of the vulva: A case report and analysis of the literature. Am J Dermatopathol 35: 685-687, 2013.

4. Ghamande SA, Kasznica J, Griffiths CT, Finkler NJ and Hamid AM: Mucinous adenocarcinomas of the vulva. Gynecol Oncol 57: 117-120, 1995.

5. Cormio G, Carriero C, Loizzi V, Gissi F, Leone L, Putignano G, Resta L and Selvaggi L: 'Intestinal-type' mucinous adenocarcinoma of the vulva: A report of two cases. Eur J Gynaecol Oncol 33: 433-435, 2012.

6. Geisler JP, Manahan KJ and Buller RE: Neoadjuvant chemotherapy in vulvar cancer: Avoiding primary exenteration. Gynecol Oncol 100: 53-57, 2006.

7. Mendoza S and Helwig EB: Mucinous (adenocystic) carcinoma of the skin. Arch Dermatol 103: 68-78, 1971.

8. Rahilly MA, Beattie GJ and Lessells AM: Mucinous eccrine carcinoma of the vulva with neuroendocrine differentiation. Histopathology 27: 82-86, 1995.

9. Graf AH, Su HC, Tubbs RR, Hacker GW, Dietz O and Staudach A: Primary neuroendocrine differentiated mucinous adenocarcinoma of the vulva: case report and review of the literature. Anticancer Res 18: 2041-2045, 1998.

10. Magrina JF, Gonzalez-Bosquet J, Weaver AL, Gaffey TA, Webb MJ, Podratz KC and Cornella JL: Primary squamous cell cancer of the vulva: Radical versus modified radical vulvar surgery. Gynecol Oncol 71: 116-121, 1998.

11. Woelber L, Trillsch F, Kock L, Grimm D, Petersen C, Choschzick M, Jaenicke F and Mahner S: Management of patients with vulvar cancer: A perspective review according to tumour stage. Ther Adv Med Oncol 5: 183-192, 2013.

12. Dittmer C, Fischer D, Diedrich K and Thill M: Diagnosis and treatment options of vulvar cancer: A review. Arch Gynecol Obstet 285: 183-193, 2012.

13. Woelber L, Mahner S, Voelker K, Eulenburg CZ, Gieseking F, Choschzick M, Jaenicke F and Schwarz J: Clinicopathological prognostic factors and patterns of recurrence in vulvar cancer. Anticancer Res 29: 545-552, 2009.

14. Domingues AP, Mota F, Durão M, Frutuoso C, Amaral N and de Oliveira CF: Neoadjuvant chemotherapy in advanced vulvar cancer. Int J Gynecol Cancer 20: 294-298, 2010.

15. Stehman FB and Look KY: Carcinoma of the vulva. Obstet Gynecol 107: 719-733, 2006.

16. Herr D, Juhasz-Boess I and Solomayer EF: Therapy for primary vulvar carcinoma. Geburtshilfe Frauenheilkd 74: 271-275, 2014. 